\title{
Non-monotonic dependence of avalanche durations on particle velocities in the discharge of a silo
}

\author{
Diego Gella ${ }^{1, *}$, Diego Maza ${ }^{1}$, and Iker Zuriguel ${ }^{1}$ \\ ${ }^{1}$ Departamento de Física y Matemática Aplicada, Facultad de Ciencias, Universidad de Navarra, Pamplona (Spain)
}

\begin{abstract}
The distributions of avalanche times between successive clog events are analyzed in a silo discharged with a conveyor belt. In a previous work [Phys. Rev. Lett. 121, 138001 (2018)], we measured the distribution of avalanche sizes (in number of particles) for the same experiment, finding a monotonous influence of both the outlet size and the velocity of particles in the clogging probability. Nonetheless, if avalanche durations are analyzed instead of avalanche sizes, a minimum is observed when representing the mean avalanche time as function of the velocity of particles. This phenomenon is explained using kinematic arguments, which are validated by experimental data. At the same time, this work aims at highlighting the importance of discerning between measuring clogging avalanches in terms of times or doing it in terms of number of particles.
\end{abstract}

\section{Introduction}

Clogging is a phenomenon that emerges in the field of granular media due to their discrete properties. Specifically, when grains pass through a bottleneck only few times bigger than the typical particle size, the aperture may be blocked by the formation of an arch (in 2D) or a dome (in 3D). The research on this problem started with the work carried out by To et al. [1], in which the process of clogging was characterized by means of a random walker model with some constraints. Later, Zuriguel et al. [2] measured the distributions of avalanche sizes $s$ (amount of material discharged between two clog events) in a granular silo discharged freely through an orifice at its bottom. The exponential trends obtained suggested a Poissonian behaviour, and the probability of finding an avalanche of size $s$ was therefore described by the following expression [3]:

$$
P(s)=p^{s}(1-p)
$$

where $p$ is the probability that a single bead goes through the orifice without forming a clogging arch. Further on, Zuriguel et al. [4] investigated the influence of the orifice size in the clogging features, discovering a dramatic growth of the mean avalanche size $\langle s\rangle$ with the orifice size $D$. The origin of this behaviour is still an open question in this field. Indeed, some authors $[4,5]$ attributed the strong rise of $s$ with $D$ to the existence of a critical orifice size above which clogging never takes place, while others [69] did it by making use of a non-divergent expression. In any case, all these works agreed on the crucial role of the outlet size in clogging development, suggesting that this is primarily a geometric problem.

*e-mail: dgella@unav.es

A video is available at https://doi.org/10.48448/650t-j118
The influence of the velocity of particles on clogging, however, has not received so much attention until recent times. This effect was firstly investigated by Arévalo et al. $[10,11]$ through changing the effective acceleration of gravity in numerical simulations. Interestingly, they obtained a finite value of the mean avalanche size when the effective gravity approached zero, but the influence of this effect was not so strong in comparison to the one of the orifice size.

Lately, we wondered whether there could be a hidden influence of the velocity of particles in the steep dependence of clogging probability on $D$. In fact, if the orifice size is modified in a free discharge, the configuration of stable arches able to block the aperture will change, but so will the velocity at which the grains cross the orifice (since it scales as $v \propto \sqrt{g D}$ [12]). To go in depth into this question, we measured the avalanche distributions in a $2 \mathrm{D}$ experimental silo in which the velocity of particles at the exit was controlled through a conveyor belt placed just below the orifice [13]. In that work, we proposed an expression for the clogging probability $p_{c}=1-p$ which decoupled the effect of the geometric and kinematic influences in the clogging process.

$$
p_{c}=(a+b v)^{-\left(D / d_{p}\right)^{2}}
$$

Here, $v$ is the typical vertical velocity of the beads crossing the exit, $d_{p}$ is the diameter of particles, while $a$ and $b$ are two fitting parameters. In particular, $a$ determines the clogging probability when the velocity of particles are reduced to the minimum, as long as $b$ accounts for the influence of $v$ in $p_{c}$.

In this work, we keep on studying the features of this system and the relationship between kinematics and clogging. Particularly, we analyze the distributions of avalanche durations $t$ (i.e. the time that passes between 
two clog events) for the same experiment than in Ref. [13]. Although in principle both magnitudes (avalanche size and duration) could seem equivalent, their relationship with the kinematics is apparently different, as it will be pointed out below. By means of analytical expressions supported by experimental data, we will connect both magnitudes and explain the origin of that presumingly different behaviour.

\section{Experimental Setup}

The experimental setup is similar to the one used in Ref. [13]. Fig. 1 displays front (a) and rear (b) photographs of it. The system consists of a two-dimensional silo built with two vertical, parallel and transparent sheets of laminated safety glass. Between them, and playing the role of silo walls, there are two aluminium plates of thickness $T=$ $0.4 \mathrm{~cm}$ supplemented by thin pieces of cardboard. Then, only a monolayer of granular material (monodisperse AISI 420 stainless steel spheres of diameter $d_{p}=0.4 \mathrm{~cm}$ ) is able to fit inside the container. Neglecting the thickness of the cardboard pieces, the available space in the silo has dimensions of $160 \times 61.2 \times 0.4 \mathrm{~cm}^{3}$. At the bottom of the silo there are two wedge-shaped pieces of stainless steel, whose separation can be changed and defines the orifice size $D$. Note that the width of the aperture in the y-direction is constant and coincides with the separation of the glasses and the particle size $\left(T=d_{p}=0.4 \mathrm{~cm}\right)$. Below the orifice there is a conveyor belt which can operate in a range of velocities $v_{b}$ between 0.1 and $16 \mathrm{~cm} / \mathrm{s}[14]$. It can be monitored from a PC thanks to its electronic controller integrated in an internal drive. The distance between the upper protrusions of its honeycomb rubber pattern and the bottom of the silo has been set in $h=3.2 \pm 0.1 \mathrm{~mm}$. In order to measure avalanche statistics, the silo is complemented with a hopper at the top to pour the grains inside it, a camera to detect the arches, a vibrator to destroy them and a LED panel.

The routine to measure the avalanche statistics is totally automated and monitored via Labview. Once the silo is filled, the conveyor belt starts running at the desired velocity. Meanwhile, the camera is acquiring monochrome images of the orifice region like the one displayed in Fig. 2 (c). If the average of the pixels in the red rectangle keeps above a predefined threshold (the box is white) during 4 seconds, the conveyor belt stops moving. Then, the avalanche duration is registered as the time lapse between the moment at which the belt switches on and the instant at which the arch is formed (when the rectangle turns white). Finally, the vibrator destroys the arch and the belt starts running again. The process is repeated as many times is required (typically 1000 avalanches per experimental condition to ensure statistical significance).

Additionally, a high-speed camera may be installed in front of the silo, with which we have taken videos at a frame rate between 125 and $500 \mathrm{fps}$. From them, we have obtained the centroids and velocities of particles at each time, and computed kinematic variables at the exit region such as the flow rate, the mean velocity of particles and the volume fraction.

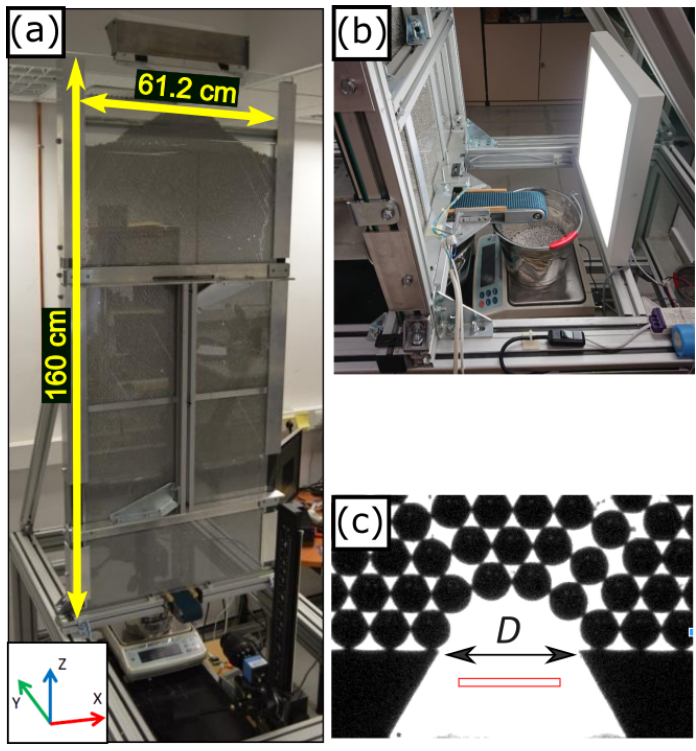

Figure 1. Photographs of the (a) front and (b) rear view of the experimental setup used in this work. (c) Monochrome image of an arch captured with the camera. The red rectangle is the area where the average value of the pixels is computed to detect the clogs and to calculate the avalanche durations.

In this work, we have performed experiments of avalanche durations statistics and computed kinematic variables for three values of the orifice sizes $(D=$ $1.33,1.53$ and $1.70 \mathrm{~cm}$ ), covering the whole range of belt velocities in each case.

\section{Results}

The survival functions of the avalanche durations for an outlet size of $D=1.53 \mathrm{~cm}\left(D / d_{p}=3.82\right)$ and seven values of the belt velocity $v_{b}$ are represented in Fig. 2. The experimental values of $v=\left|\left\langle v_{z}\right\rangle\right|$, the absolute value of the mean vertical velocity of the particles, have been also included in the legend since this magnitude is the representative one to characterize the velocity of discharge as reported in Ref. [13]. Notice also that the sets of curves for the other two orifice sizes explored (not shown) present similar features.

Clearly, all curves follow exponential trends (note the semi-logarithmic scale in the y-axis), suggesting a Poissonian behaviour like the one presented by the avalanche sizes in the same experiment [13]. However, the evolution of the negative slopes of the curves shows an intriguing phenomenon as $v$ increases. Starting from moderate values at low $v$, the curves become steeper (and the slopes larger) until intermediate values of $v$ around $\sim 6 \mathrm{~cm} / \mathrm{s}$. At this point, the trend changes and for the highest values of $v$ the slopes diminish and avalanche durations become longer. This behaviour can be appreciated in a more visual way in Fig. 3, where the mean avalanche times are represented for three different outlet sizes as function of $v$. In all cases, the mean avalanche durations present a non-monotonic behaviour as function of particles velocity. The curves exhibit a minimum at a value of $v$ which appears to shift towards smaller values of $v$ as the aspect 


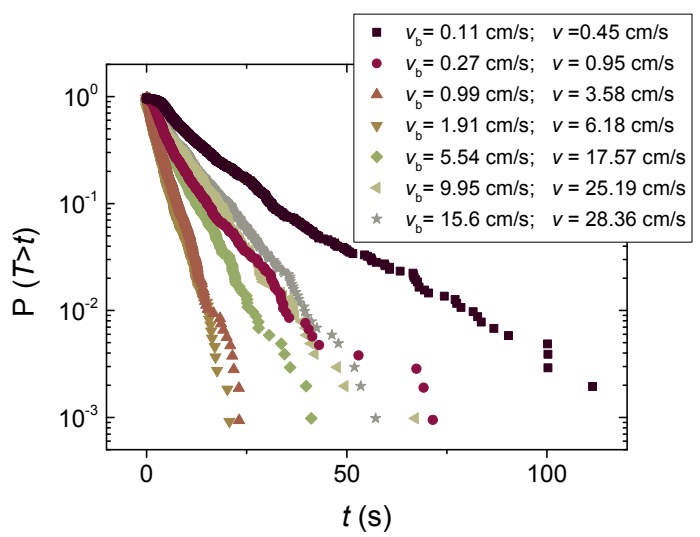

Figure 2. Experimental survival functions of the avalanche durations for an outlet size of $D=1.53 \mathrm{~cm}\left(D / d_{p}=3.82\right)$ and the typical particle velocities displayed in the legend.

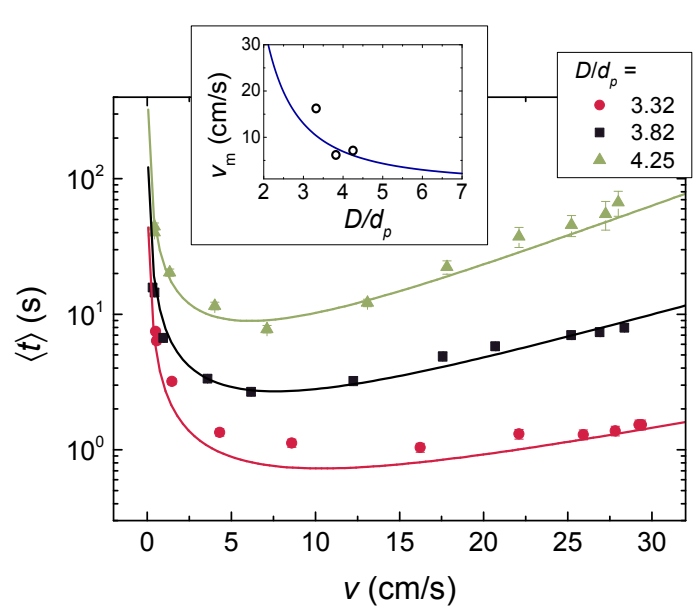

Figure 3. Experimental mean avalanche durations as function of the velocity of beads for the three aspect ratios shown in the legend. The solid lines are plots of Eq. 7 with the values of the parameters indicated in the text. The solid line in the inset is a plot of Eq. 8, which relates the beads velocities at which the mean avalanche duration is minimum with respect to $D / d_{p}$. The empty circles are experimental values of $v$ for which $\langle t\rangle$ is minimum for the three values of $D / d_{p}$ explored in this work.

ratio is enlarged. Furthermore, for small values of $v$ the mean avalanche times seem to dramatically increase as $v$ approaches zero, while for high particle velocities a considerable growth is also observed.

Now, we are going to interpret these results by making use of the expressions introduced above. If we compute $\langle s\rangle$ as the first moment of Eq. 1 and consider that the avalanches measured involve an amount of grains significantly greater than 1 , we can write:

$$
\langle s\rangle=\frac{1}{p_{c}}-1 \simeq p_{c}^{-1}=(a+b v)^{\left(D / d_{p}\right)^{2}}
$$

Assuming that the flow rate in an avalanche is reasonably steady, $\langle s\rangle$ can be related to $\langle t\rangle$ by means of the particle flow rate $W_{b}$ :

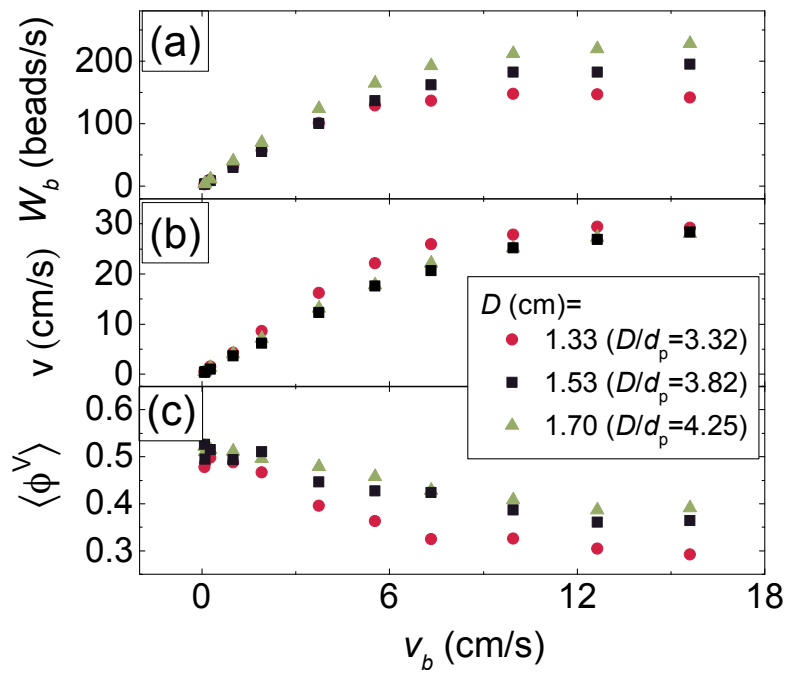

Figure 4. Experimental values of (a) the module of the mean vertical velocity (b) the particle flow rate and (c) the volume fraction, as function of the belt velocity for the three outlet sizes displayed in the legend.

$$
W_{b}=\frac{\langle s\rangle}{\langle t\rangle}
$$

Combining Eqs. 3 and 4, the expression results:

$$
\langle t\rangle=\frac{(a+b v)^{\left(D / d_{p}\right)^{2}}}{W_{b}}
$$

The non-monotonicity of the mean avalanche times as function of $v$ can be explained from an analysis of Eq. 5 in the limits of low and high particle velocities. To do this, let us start by looking at the dependences of the experimental values of $W_{b}$ and $v$ on the belt velocity $v_{b}$, which are represented in Fig. 4 (a) and (b). The evolution is similar in both cases for the three outlet sizes studied: they exhibit a linear behaviour for low values of $v_{b}$ and a saturation in a constant value ( $D$-dependent in the case of $W_{b}$ ). Furthermore, both magnitudes tend to zero when the belt velocity is decreased to zero. Therefore, the denominator of Eq. 5 tends to zero when $v$ is reduced to the minimum. The divergence observed at $v \rightarrow 0$ is then explicated by the fact that at this limit of the velocities the numerator tends to a non-zero (and $D$-dependent) value. In the other extreme, for big values of $v$ the growth of the numerator is considerable higher than the one of the denominator (which indeed tends to saturate), leading also to large values of $\langle t\rangle$ for increasingly high $v$ values. In consequence, if we consider a continuous and differentiable function $\langle t\rangle(v)$ in the interval $(0, \infty)$, necessarily there will be a closed interval $\left[v_{\alpha}, v_{\beta}\right] \in(0, \infty)$ such that $\langle t\rangle\left(v_{\alpha}\right)=\langle t\rangle\left(v_{\beta}\right)$. Therefore, Rolle's theorem claims that there must be a $v_{\gamma} \in\left[v_{\alpha}, v_{\beta}\right]$ such that $\langle t\rangle^{\prime}\left(v_{\gamma}\right)=0$, which can only be a minimum in this case.

Indeed, Eq. 5 can be expressed as function of $v$ by relating it with the particle flow rate $W_{b}$ in the spirit of Ref. [15]: 


$$
W_{b}=\frac{\left\langle\phi^{V}\right\rangle T D v}{V_{p}}
$$

where $V_{p}$ is the volume of one particle, $\left\langle\phi^{V}\right\rangle$ is the mean volume fraction of the grains crossing the outlet and $T=$ $d_{p}$ is the outlet dimension in the y-direction (hence $T D$ accounts for the outlet section). Substituting Eq. 6 in Eq. 5 reads:

$$
\langle t\rangle=\frac{V_{p}}{\left\langle\phi^{V}\right\rangle T D} \frac{(a+b v)^{\left(D / d_{p}\right)^{2}}}{v}
$$

It is worth to mention that in this new expression, all parameters are known and independent on $v$ except perhaps $\left\langle\phi^{V}\right\rangle$. The relationship between these two variables can be figured out in Fig. 4 (c), where the experimental data of the volume fraction are represented as function of $v_{b}$. As it is observed there, this magnitude decreases when the outlet size is reduced or the velocity of particles increased. Nevertheless, we will assume for sake of simplicity that $\left\langle\phi^{V}\right\rangle$ is weakly dependent on $v$ and therefore we will consider that Eq. 7 is a function of only one variable: the velocity of particles. In particular, the average value of all $\left\langle\phi^{V}\right\rangle$ data, $\left\langle\overline{\phi^{V}}\right\rangle=0.44$ is used. Then, the values of $\langle t\rangle$ obtained from Eq. 7 are compared to the experimental ones in Fig. 3. Remarkably, the curves for the three different outlet sizes studied describe nicely the experimental data using the values of the parameters $a$ and $b$ reported in Ref. [13] $\left(a=1.33\right.$ and $\left.b=0.0128(\mathrm{~cm} / \mathrm{s})^{-1}\right)$ [16].

Additionally, the shift of the position of the minima of the curves in Fig. 3 can be computed as

$$
\frac{d\langle t\rangle}{d v}=0 \Longrightarrow v_{m}=v\left[\langle t\rangle=\langle t\rangle_{\min }\right]=\frac{a}{b} \frac{1}{\left(D / d_{p}\right)^{2}-1}
$$

According to this simple result, the velocities at which $\langle t\rangle$ is minimum exhibit an inverse dependence on the square of the aspect ratio weighted with the ratio between the parameters $\mathrm{a}$ and $\mathrm{b}$. It should be reminded that $a$ is a parameter that determines the clogging probability when the grains velocity is negligible (geometrical contribution) while $b$ gather the influence of the velocity of particles [13]. To provide a visual perception of this formula, a plot of Eq. 8 as function of the aspect ratio has been included in the inset of Fig. 3 for the case studied here. The three experimental values of $v$ for which $\langle t\rangle$ is minimum in the main panel of Fig. 3 have been also included in that inset. These data, represented with empty circles, suggest to follow a similar decreasing trend than the one Eq. 8 .

\section{Conclusions}

In this work, the existence of a minimum in the dependence of the mean avalanche duration on particles velocity has been reported. The knowledge of this phenomenon could be significant in industrial fields, allowing to avoid operating in certain range of parameters if silos are discharged in a controlled way using conveyor belts.

Still, it must be mentioned that the nature of this phenomenon is not a novelty per se. Indeed, the minimum arises only from the relationship between the dependences of the mean avalanche size and the particle flow rate on the velocity of particles, and from the fact that there is a non-zero limit for $\langle s\rangle$ when $v$ approaches zero.

Finally, we would like to remark that the election of either avalanche sizes or avalanche durations as a magnitude to measure the clogging features in a system is not equivalent. As it has been observed here, their behaviour is significantly different when the kinematic properties of the system are varied and this could lead to mistakes if this aspect is not taken into account.

\section{Acknowledgements}

This work was funded by Ministerio de Economía y Competitividad (Spain) through Projects No. FIS201457325 and No. FIS2017-84631 MINECO/AEI/FEDER, UE Project.

\section{References}

[1] K. To, P.-Y. Lai and H.K. Pak, Phys. Rev. Lett. 86 (1), 71 (2001).

[2] I. Zuriguel, L.A. Pugnaloni, A. Garcimartín and D. Maza, Phys. Rev. E 68, 030301(R) (2003).

[3] I. Zuriguel, PhD Thesis: Flujo y atascos de un medio granular en la descarga de silos (Universidad de Navarra, Pamplona, 2005).

[4] I. Zuriguel, A. Garcimartín, D. Maza, L.A. Pugnaloni and J.M. Pastor, Phys. Rev. E 71, 051303 (2005).

[5] T. Masuda, K. Nishinari and A. Schadschneider, Phys. Rev. Lett. 12, 138701 (2014).

[6] K. To, Phys. Rev. E 71, 060301(R) (2005).

[7] A. Janda, I. Zuriguel, A. Garcimartín, L.A. Pugnaloni and D. Maza, EPL 84, 44002 (2008).

[8] C.C. Thomas and D.J. Durian, Phys. Rev. Lett. 114, 178001 (2015).

[9] A. Marin, H. Lhuissier, M. Rossi and C.J. Kähler, Phys. Rev. E 97, 021102 (R) (2018).

[10] R. Arévalo, I. Zuriguel, D. Maza and A. Garcimartín, Phys. Rev. E 89, 042205 (2014).

[11] R. Arévalo and I. Zuriguel, Soft Matter 12, 123-130 (2016).

[12] A. Janda, I. Zuriguel and D. Maza, Phys. Rev. Lett. 108, 248001 (2012).

[13] D. Gella, I. Zuriguel and, D. Maza, Phys. Rev. Lett. 121, 138001 (2018).

[14] The range of $v_{b}$ expressed in a dimensionless way, $v_{b}^{*}=v_{b} / \sqrt{g d_{p}}$ (where $\sqrt{g d_{p}}$ is the velocity that a particle reaches when falls a distance equal to its own radius by action of gravity) covers between 0.005 and 0.8 .

[15] D. Gella, D. Maza and I. Zuriguel, Powder Technol. 360, 104-111 (2020).

[16] It is possible to build a dimensionless variable $b^{*}=$ $b * \sqrt{g d_{p}}$. The result would be $b^{*}=0.25$, a value which is lesser than the one obtained for $a$. 